\title{
Improved Fabrication Method for Porous PMMA-titania Composite Photocatalytic Materials
}

Justin D. Glover ${ }^{1}$, Autumn G. Horne ${ }^{1}$, Jeron D. Crawford ${ }^{1}$, and Joel E. Boyd ${ }^{12^{\star}}$

${ }^{1}$ Department of Chemistry, Erskine College, Washington St, Due West, SC 29639, USA

${ }^{2}$ Center for Environmental Stewardship, Erskine College, Washington St, Due West, SC 29639, USA

\section{Abstract}

Porous poly(methyl methacrylate) (PMMA) infused with commercially prepared titania nanoparticles has been previously investigated for photocatalytic applications. The benchmark method of making this material involves introducing an aqueous dispersion of titaniainto a solution of PMMA, acetone, and TWEEN surfactant. This process leads to the coagulation of a porous PMMA/titania material with substantial photocatalytic activity. Revising the fabrication process to incorporate the titania directly into the PMMA-acetone solution prior to coagulation with distilled water, produces porous composite photocatalytic materials that use less $\mathrm{TiO}_{2}$ to achieve comparable photocatalytic degradation rates, while also reducing the titania loss during photocatalytic use of the composite. These new materials are thus more attractive candidates for photocatalytic applications, especially where the release of nanoscale titania is undesirable.

\section{Publication History:}

Received: April 16, 2017 Accepted: July 01, 2017

Published: July 03, 2017

\section{Keywords:}

$\mathrm{TiO}_{2}$, Photocatalysis, Green photocatalysis factor, Green chemistry

\section{Introduction}

The photocatalytic activity of metal oxide photocatalysts such as titania $\left(\mathrm{TiO}_{2}\right)$ is greatly enhanced through the use of nanoscale rather than bulk materials. Although post-use removal of nanoparticulate photocatalysts is not impossible, it at the very least adds an additional time consuming complication to the photocatalytic purification of water [1]. For this reason, composite photocatalytic materials where $\mathrm{TiO}_{2}$ is deposited on or contained within a macroscopic support material have been extensively investigated [1-11]. UV transparent supports are beneficial in that they facilitate reactor designs where the activating light can be delivered either through the contaminated water, or directly through the support, avoiding the need for the light to traverse the water within the reactor system. This can become important in situations where the aqueous solution absorbs the wavelength of light used by the photocatalyst. Porous support materials are also advantageous in that they increase the surface area of available photocatalyst, and help retain the photocatalyst during use. Stewart et al. recently reported a porous PMMA-titania composite material fabricated by coagulating a PMMA-acetoneTWEEN solution in an aqueous dispersion of $\mathrm{TiO}_{2}$ [5]. In that paper, the Green Photocatalysis Factor (GPF) shown below in equation 1 was introduced as a metric that simultaneously reflects the photocatalytic activity of the composite, and the amount of $\mathrm{TiO}_{2}$ lost from the composite during use.

$$
G P F=\frac{k\left(\mathrm{~min}^{-1}\right)}{\text { TiO }_{2} \text { mass lost }(g)}
$$

Since the effects of nanoscale $\mathrm{TiO}_{2}$ on human health and the environment is not yet a resolved issue, it is important to minimize the release of $\mathrm{TiO}_{2}$ during photocatalytic applications [12-15]. The GPF takes this concern into consideration and divides the degradation rate constant by the amount of $\mathrm{TiO}_{2}$ released from the sample [5].

The PMMA-titania samples fabricated by Stewart et al. were freestanding disks with a thickness of $1 \mathrm{~cm}$ transverse to the flow of water through the porous wafer. Although these materials do exhibit high photocatalytic activity, nearly all of that activity is due to $\mathrm{TiO}_{2}$ very near to the sample surface. Figure 1 is a photograph of a PMMA-

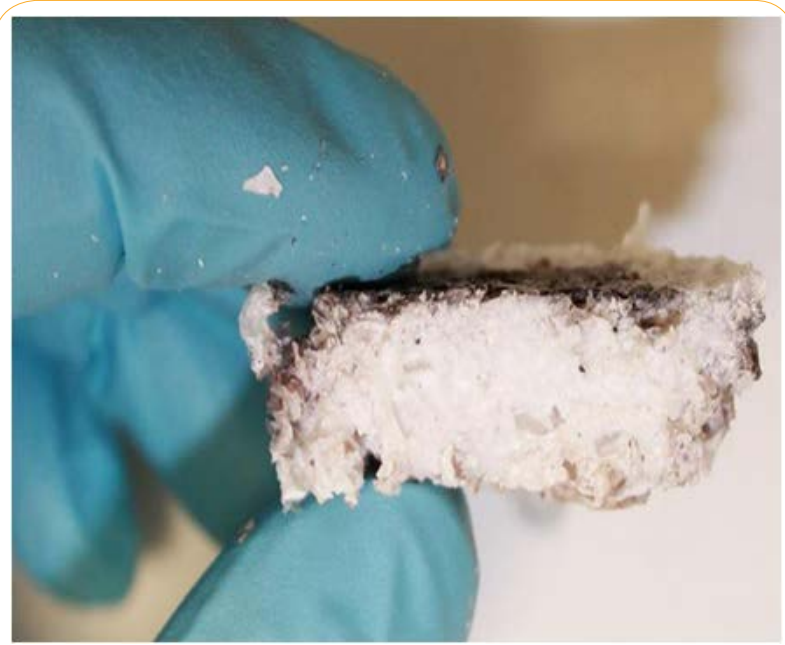

Figure 1: Cross-section of a porous PMMA-titania wafer fabricated with the benchmark method. The dark surface color is the result of photodeposited Ag when illuminated from above in aqueous $\mathrm{AgNO}_{3}$.

titania sample fabricated using the Stewart method which was then used to photocatalytically reduce $\mathrm{Ag}^{+}$from a $0.1 \mathrm{M}$ aqueous $\mathrm{AgNO}_{3}$ solution containing methanol in 3-fold stoichiometric excess. The UV irradiance was provided from above the sample, and the Ag was photodeposited exclusively at the sample surface. Thick samples such as this contain substantial amounts of $\mathrm{TiO}_{2}$ within the sample core that is not photocatalytically active, but is still prone to loss from the composite during use as water flows through the porous membrane. Due to the poor mechanical integrity of thin porous PMMA films,

${ }^{*}$ Corresponding Author: Prof. Joel E. Boyd, Department of Chemistry, Erskine College and Center for Environmental Stewardship, 2 Washington St, Due West, SC 29639, USA; E-mail: boyd@erskine.edu

Citation: Glover JD, Horne AG, Crawford JD, Boyd JE (2017) Improved Fabrication Method for Porous PMMA-titania Composite Photocatalytic Materials. Int J Metall Mater Eng 3: 135. doi: https://doi.org/10.15344/2455-2372/2017/135

Copyright: (c) 2017 Glover et al. This is an open-access article distributed under the terms of the Creative Commons Attribution License, which permits unrestricted use, distribution, and reproduction in any medium, provided the original author and source are credited. 
Citation: Glover JD, Horne AG, Crawford JD, Boyd JE (2017) Improved Fabrication Method for Porous PMMA-titania Composite Photocatalytic Materials. Int J Metall Mater Eng 3: 135. doi: https://doi.org/10.15344/2455-2372/2017/135

Page 2 of 5

samples thinner than $0.5 \mathrm{~cm}$ require deposition on a solid surface such as non-porous PMMA slab. In this work, $0.38 \mathrm{~mm}$ thick samples fabricated through the benchmark method [5] are compared to a revised fabrication method in which the titania is included within the PMMA-acetone-TWEEN solution, rather than in the coagulating water. The result is a material that has comparable photocatalytic activity while both using less $\mathrm{TiO}_{2}$ during fabrication and losing less $\mathrm{TiO}_{2}$ during long term usage.

Antibacterial $\mathrm{TiO}_{2}$-based photocatalytic extruded plastic films have been previous reported, which are similar to these PMMA-titania composites in that they have $\mathrm{TiO}_{2}$ mixed throughout the polymeric material. These extruded plastic films have shown evidence of increased activity after exposure to UV light [16]. This is due to an increase in the amount of exposed $\mathrm{TiO}_{2}$ on the surface of the material due to the photocatalytically oxidation of the support material by the $\mathrm{TiO}_{2}$ embedded within it. This preparatory UV exposure increased the exposed $\mathrm{TiO}_{2}$ after a $48 \mathrm{~h}$ preparative UV exposure. The photocatalytic activity of each sample was evaluated as the pseudo first order rate constant in the decolorization of $10 \mathrm{ppm}$ methyl orange. The durability of these materials is evaluated by comparing the photocatalytic activity before and after a $1000 \mathrm{~h}$ period of continual UV exposure. The GPF is used as a metric for material comparison and the results are interpreted in view of the use of these materials in a long-term sustainable water purification application.

\section{Materials and Method}

\section{Materials}

Degussa/Evonik P-25 with an average surface area of $50 \mathrm{~m}^{2} / \mathrm{g}$ and an average particle size of $30 \mathrm{~nm}$ was the nanoscale $\mathrm{TiO}_{2}$ used. ACS grade acetone was purchased from Fisher Scientific along with TWEEN 80 surfactant. PMMA (molar mass of 75,000 g/mol) was purchased from Polysciences Inc. UV-transparent acrylic (PolycastSolacryl SUVT) with a thickness of $4.76 \mathrm{~mm}$ was used as the support material, and in order to control film thickness, a lab-built doctor's blade was fabricated and calibrated with steel reference spacers. An ultraviolet light-emitting diode (UV-LED) lamp with $\lambda_{\text {max }}=365 \mathrm{~nm}$ was purchased from Xenopus Electronix (XeLED-Ni1UV-R4-365-E27-SS) and used for all degradation experiments. $13 \mathrm{~W}$ UV-compact fluorescent bulbs were used for the burn in and long-term UV exposure experiments.

\section{Sample fabrication}

All samples were fabricated and evaluated in triplicate. For the fabrication of the materials using the benchmark method, $1.8 \mathrm{~g}$ of PMMA was added to $10 \mathrm{~mL}$ of acetone followed by $0.21 \mathrm{~g}$ of TWEEN 80. This solution was applied over a $6.4 \times 7.6 \mathrm{~cm}$ nonporous acrylic area and spread to a $0.38 \mathrm{~mm}$ thick coating using a lab-built doctor's blade. The non-porous acrylic support slab surface was pre-roughened by the manufacturer, facilitating the adhesion of the porous PMMA-titania composite film. Immediately after the PMMA film was deposited, $10 \mathrm{~mL}$ of an aqueous dispersion of $\mathrm{TiO}_{2}$ was then sprayed over the solution with a hand-operated spray bottle. The coagulating solution concentration was varied, using the following concentrations: $1,5,10$, and $15 \%(\mathrm{w} / \mathrm{v}) \mathrm{TiO}_{2}$. The resulting samples were dried overnight at $23^{\circ} \mathrm{C}$ and then washed by pouring $2 \mathrm{~L}$ of distilled water gently over the porous surface. The $\mathrm{TiO}_{2}$ content for each of the benchmark sample was determined by subtracting the average weight of samples that did not contain $\mathrm{TiO}_{2}$ from each sample that contained $\mathrm{TiO}_{2}$.
In order to make the revised samples, $10 \mathrm{~mL}$ of $15,30,44$, or 56 $\%(\mathrm{w} / \mathrm{w}) \mathrm{TiO}_{2} /(\mathrm{PMMA})$ was mixed with $10 \mathrm{~mL}$ of acetone and $0.21 \mathrm{~g}$ of TWEEN 80. The resulting solution was applied to a $6.4 \mathrm{~cm} \times 7.6 \mathrm{~cm}$ nonporous acrylic area and spread to a film thickness of $0.38 \mathrm{~mm} .10 \mathrm{~mL}$ of distilled water was then sprayed over the solution. The resulting samples were dried overnight at $23^{\circ} \mathrm{C}$ and then washed by pouring $2 \mathrm{~L}$ of distilled water over the porous surface. The $\mathrm{TiO}_{2}$ content in each of the revised samples was determined from the difference of the weight of the acrylic slide before and after application, multiplied by the percent $\mathrm{TiO}_{2} / \mathrm{PMMA}$ used.

\section{Photocatalytic testing}

Photocatalytic testing was performed with each sample mounted at $34^{\circ}$ from horizontal. A UV LED lamp was placed $17.8 \mathrm{~cm}$ above the sample surface. The irradiance at the sample surface was $8 \mathrm{~mW} /$ $\mathrm{cm}^{2}$, and was provided perpendicular to the sample surface. Using a peristaltic pump, $75 \mathrm{~mL}$ of 10 ppm methyl orange was continually recirculated across the sample surface as shown in Figure 2. The methyl orange flowed down the entire porous surface at a rate of $200 \mathrm{~mL} / \mathrm{min}$. The absorbance of the methyl orange at $463 \mathrm{~nm}$ was collected as a function of time over a period of $30 \mathrm{~min}$. The pseudo first order rate constant determined from this data was used as the metric for photocatalytic activity of each sample.

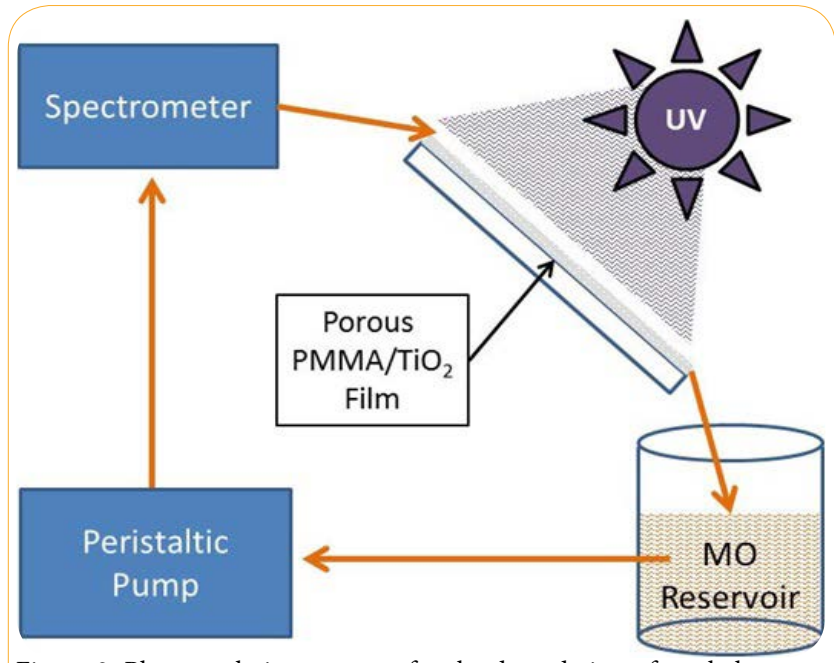

Figure 2: Photocatalytic apparatus for the degradation of methyl orange (MO).

\section{Surface characterization and preparation}

Scanning electron microscopy: SEM images of both a benchmark sample and a revised-method sample were captured using a variable pressure scanning electron microscope (Hitachi SU660).

Preliminary UV exposure: SP reliminary UV exposure: In order to maximize the amount of exposed $\mathrm{TiO}_{2}$ on the sample surface, each sample was exposed to UV light prior to testing. The preparative UV exposure involved horizontally submerging the sample in a distilled water bath with the $\mathrm{TiO}_{2}$ film facing upwards. A compact UV fluorescent bulb was centered $5.1 \mathrm{~cm}$ above each slide and $3.8 \mathrm{~cm}$ above the water surface, providing illumination at $2.5 \mathrm{~mW} / \mathrm{cm}^{2}$. To determine the optimal UV exposure time, three benchmark samples containing $0.129 \pm 0.093 \mathrm{~g}$ of $\mathrm{TiO}_{2}$, and three revised-method samples, containing $0.113 \pm 0.020 \mathrm{~g}$ of $\mathrm{TiO}_{2}$ were exposed for $72 \mathrm{~h}$. Every $12 \mathrm{~h}$, the photocatalytic activity of each sample was determined. After each testing cycle, the samples were rotated to a different bulb within the reservoir to homogenize average UV exposure.

Int J Metall Mater Eng

ISSN: 2455-2372

IJMME, an open access journal Volume 3. 2017. 135 
Citation: Glover JD, Horne AG, Crawford JD, Boyd JE (2017) Improved Fabrication Method for Porous PMMA-titania Composite Photocatalytic Materials. Int J Metall Mater Eng 3: 135. doi: https://doi.org/10.15344/2455-2372/2017/135

Page 3 of 5

Methylene blue adsorption: The exposed $\mathrm{TiO}_{2}$ surface area of each sample type was evaluated using a methylene blue adsorbance test modified from Ratova et al. [16]. Each sample was completely submerged in approximately $520 \mathrm{~mL}$ of a stirred $100 \mathrm{ppm}$ methylene blue/ $\mathrm{NaOH}(\mathrm{pH} 11)$ aqueous solution for $1 \mathrm{~h}$. The $\mathrm{NaOH}(\mathrm{pH} 11)$ causes the $\mathrm{TiO}_{2}$ surface to take on a negative charge, thus attracting the cationic methylene blue. The sample was then rinsed repeatedly with $150 \mathrm{~mL}$ of aqueous $\mathrm{NaOH}(\mathrm{pH} \mathrm{11).} 4$ or 5 rinses of $150 \mathrm{~mL}$ were needed to receive a colorless rinse solution. After rinsing, the slides were suspended in $520 \mathrm{~mL}$ of $\mathrm{HCl}(\mathrm{pH} 3)$ for $1 \mathrm{~h}$ to remove the adsorbed methylene blue. The samples were then rinsed twice with $150 \mathrm{~mL}$ of $\mathrm{HCl}$, and the combined $\mathrm{HCl}$ soak and wash solution was then diluted to $1 \mathrm{~L}$ prior to UV-Vis analysis at $665 \mathrm{~nm}$. The amount of methylene blue adsorbed on each sample surface is a convenient estimation of the amount of exposed $\mathrm{TiO}_{2}$ on the sample surface [16].

\section{Durability test}

In order to evaluate the long term stability of these materials, samples were illuminated with a UV-CFL with a surface irradiance of $2.5 \mathrm{~mW} / \mathrm{cm}^{2}$ for a total of $1000 \mathrm{~h}$. Using three separate pumps, distilled water was recirculated from a common reservoir over the surface of separate triplicate samples. The slides were again placed at $34^{0}$ from horizontal with the water flowing over them at $210 \mathrm{~mL} / \mathrm{min}$. In total, six samples were tested, three benchmark samples containing $0.129 \pm 0.093 \mathrm{~g}$ of $\mathrm{TiO}_{2}$, and three revised-method samples containing $0.113 \pm 0.020 \mathrm{~g}$ of $\mathrm{TiO}_{2}$. Following this $1000 \mathrm{~h}$ period, the $\mathrm{TiO}_{2}$ content of the water was quantified using ICP-MS (PerkinElmer Elan 9000).

\section{Results and Discussion}

\section{Surface characterization and preparation}

Scanning electron microscopy: The SEM images (Figure 3) clearly show a fundamental difference between the benchmark fabrication method and the revised technique. When the $\mathrm{TiO}_{2}$ is included within the coagulating water, the $\mathrm{TiO}_{2}$ is primarily deposited on the PMMA surface in relatively thick agglomerated rafts. Inclusion of the $\mathrm{TiO}_{2}$ within the PMMA matrix itself produces composites where the $\mathrm{TiO}_{2}$ is included throughout the PMMA matrix, both within the PMMA and on the PMMA surface. The embedded $\mathrm{TiO}_{2}$ is less prone to loss from the composite surface, but also benefits from the preliminary UV exposure process to maximize the amount of $\mathrm{TiO}_{2}$ exposed for the photocatalytic interaction with the contaminated water.

Preliminary UV exposure: Figure 4 displays the pseudo first order rate constant for the decolorization of methyl orange as a function of UV exposure time for the benchmark and revised method samples containing $0.129 \pm 0.093 \mathrm{~g}$ and $0.113 \pm 0.020 \mathrm{~g}$ of $\mathrm{TiO}_{2}$ respectively. The improvement in photocatalytic activity is apparently complete for both sample types after $36 \mathrm{~h}$; and a $48 \mathrm{~h}$ UV exposure period was thus chosen for all subsequent samples.

Methylene blue adsorption: The mass of methylene blue that adsorbs on the composite surface depends on the amount of $\mathrm{TiO}_{2}$ present in the sample and the effect of the UV exposure (Figure 5). The increase in photocatalytic activity that accompanies the preparatory UV exposure is attributed to an increase in exposed $\mathrm{TiO}_{2}$ on the sample surface as the $\mathrm{TiO}_{2}$ oxidizes the polymeric matrix in which it is embedded [16]. By comparing the mass of methylene blue adsorbed before and after $48 \mathrm{~h}$ UV exposure, (Figure 5), it is evident that UV exposure enhances $\mathrm{TiO}_{2}$ surface area for samples with low
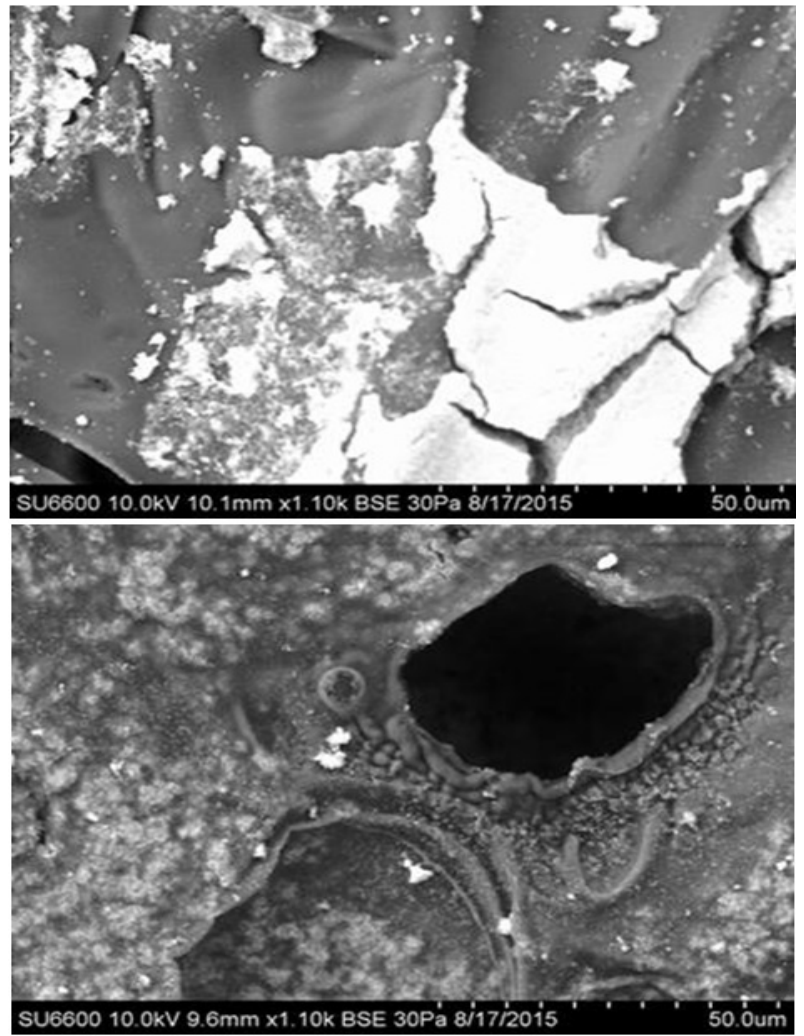

Figure 3: SEM micrographs of benchmark (top) and revised (bottom) sample. The high contrast nanoscale $\mathrm{TiO}_{2}$ as well as the embedded $\mathrm{TiO}_{2}$ is clearly seen on the revised-fabrication sample.

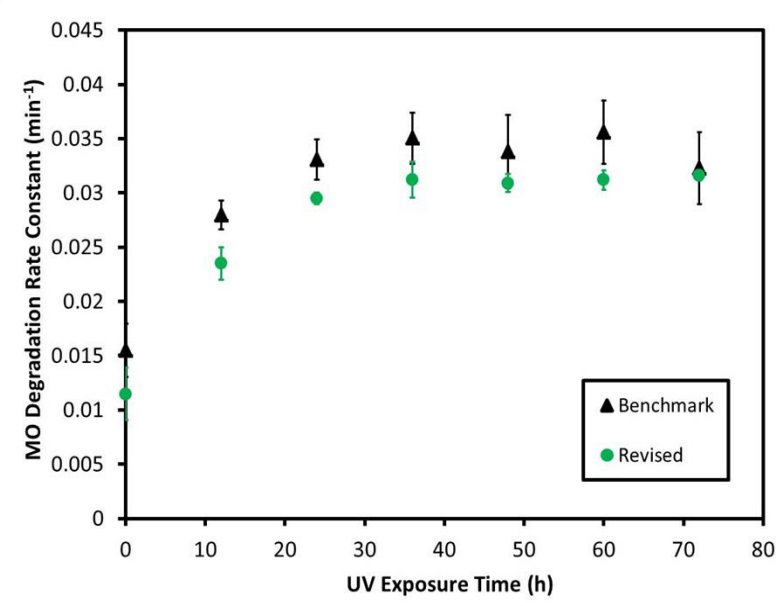

Figure 4: Methyl orange (MO) decolorization rate constant as a function of the UV exposure period for each sample type with comparable $\mathrm{TiO}_{2}$ content. The error bars represent one standard deviation from triplicate samples.

$\mathrm{TiO}_{2}$ content (up to $0.1 \mathrm{~g}$ ). This surface area enhancement is not realized in samples with higher $\mathrm{TiO}_{2}$ content presumably because the exposed $\mathrm{TiO}_{2}$ present is already maximized, and ongoing UV exposure results in the loss of $\mathrm{TiO}_{2}$ from the composite surface.

\section{Photocatalytic activity testing and $\mathrm{TiO}_{2}$ loss}

Samples of varying $\mathrm{TiO}_{2}$ content from both preparation methods were photocatalytically evaluated before and after the $48 \mathrm{~h}$ UV 


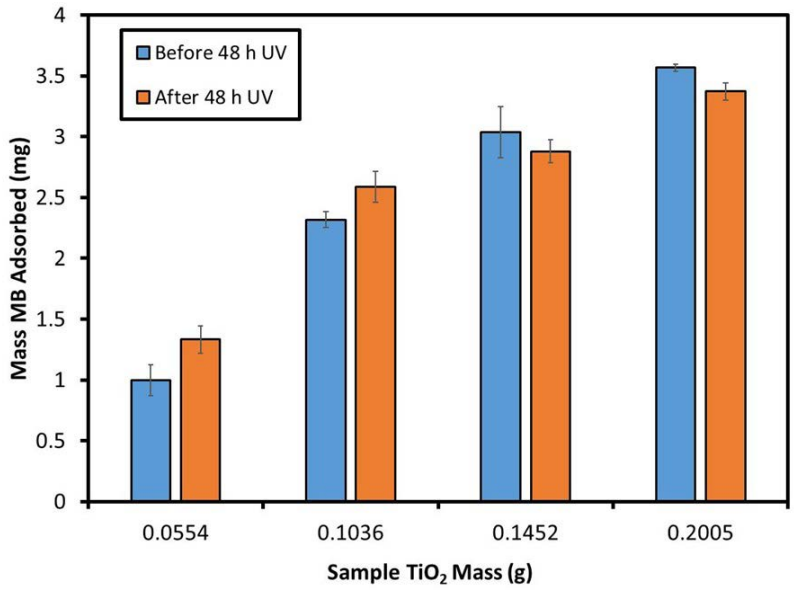

Figure 5: Mass of methylene blue (MB) adsorbed by revised fabrication samples at varying $\mathrm{TiO}_{2}$ content both before and after $48 \mathrm{~h} \mathrm{UV}$ exposure.

The error bars represent one standard deviation from triplicate samples.

of these samples. The photocatalytic activity generally increases with pre-exposure. Figure 6 shows the pseudo first order rate constants for each the amount of $\mathrm{TiO}_{2}$ available, and there is not a substantial difference in activity between the benchmark and revised samples with similar amounts of photocatalyst present. In the process of measuring the pre and post UV-exposure photocatalytic activity, each sample was exposed to $2 \mathrm{~h}$ of UV illumination at an irradiance of $8 \mathrm{~mW} / \mathrm{cm}^{2}$. The solutions used in this process were combined and analyzed for the presence of $\mathrm{TiO}_{2}$. The mass of titania lost from each sample is shown in Figure 7. There is a general and expected increase in $\mathrm{TiO}_{2}$ loss as the total $\mathrm{TiO}_{2}$ content of the sample is increased. The high $\mathrm{TiO}_{2}$ benchmark samples exhibit the most significant losses, as much of the $\mathrm{TiO}_{2}$ in these samples is present in aggregates on the sample surface as shown in the top panel in Figure 3. Since much of this $\mathrm{TiO}_{2}$ is not firmly embedded on or within the PMMA matrix, it is easily lost during use. The samples exhibiting the lowest $\mathrm{TiO}_{2}$ loss are the revised method-samples with the lowest $\mathrm{TiO}_{2}$ content.

\section{Green photocatalysis factor}

The GPF for each sample is shown in Figure 8. The samples with the highest GPF values are from the revised fabrication method, with the $0.113 \pm 0.020 \mathrm{~g}$ revised sample yielding a GPF that is $83 \%$ greater than the benchmark sample containing $0.129 \pm 0.093 \mathrm{~g} \mathrm{TiO}_{2}$. The ideal amount of $\mathrm{TiO}_{2}$ is likely an application-dependent choice. If a composite material is intended for extended-term use, or in applications such as drinking water purification where nanoscale $\mathrm{TiO}_{2}$ tolerances are low, samples with minimal $\mathrm{TiO}_{2}$ content could be preferable despite the lower photocatalytic activity that they provide. The GPF provides a case-specific tool for comparing photocatalytic materials options across platforms.

The lifetime of a photocatalytic material is of substantial interest for virtually any potential commercial or field application. To investigate the long-term durability of these materials, one triplicate set of benchmark $\left(0.129 \pm 0.093 \mathrm{~g} \mathrm{TiO}_{2}\right)$ and one triplicate set of revised method samples $\left(0.113 \pm 0.020 \mathrm{~g} \mathrm{TiO}_{2}\right)$ were exposed to UV light for $1000 \mathrm{~h}$ prior to re-testing of the photocatalytic activity of each sample and the quantification of the $\mathrm{TiO}_{2}$ loss during the $1000 \mathrm{~h}$ period. The degradation rate constant for the benchmark samples actually slightly increased by $2 \%$, and the revised-method samples decreased their activity by almost $16 \%$. In this $1000 \mathrm{~h}$ time period, the benchmark

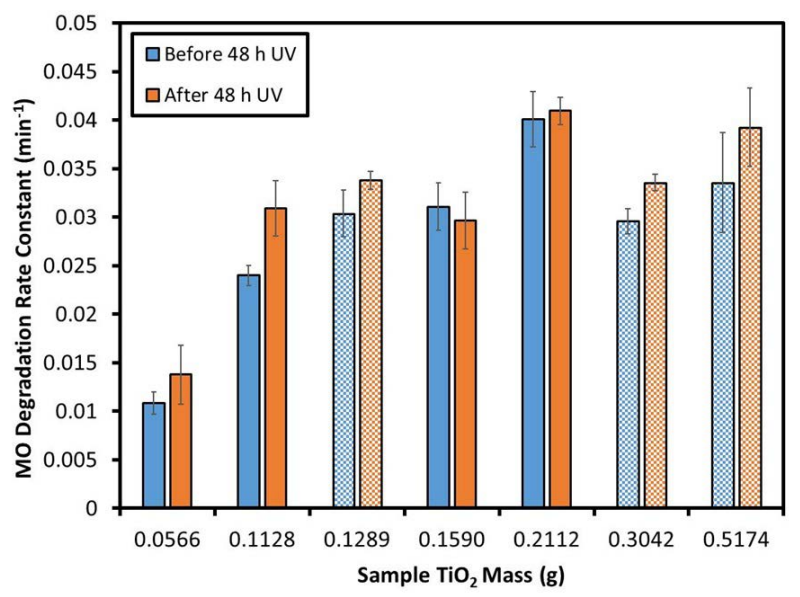

Figure 6: Pseudo first order rate constants for the photocatalytic decolorization of methyl orange (MO) before and after $48 \mathrm{~h}$ UV exposure for both benchmark samples (checkered bars) and revised-method samples (solid bars). The error bars represent one standard deviation from triplicate samples.

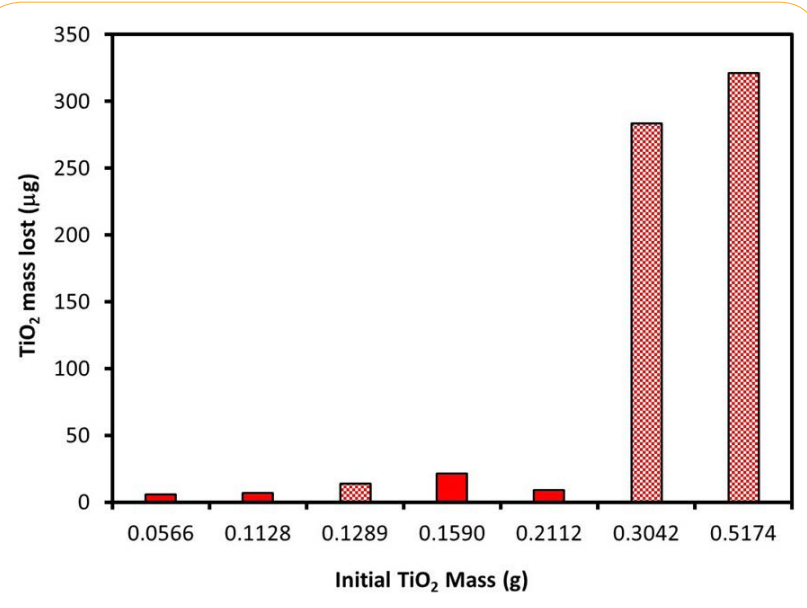

Figure 7: The mass of titania lost from each sample type during the $2 \mathrm{~h}$ initial photocatalytic use of each sample type. Checkered bars represent benchmark samples, and solid bars represent revised-fabrication samples.

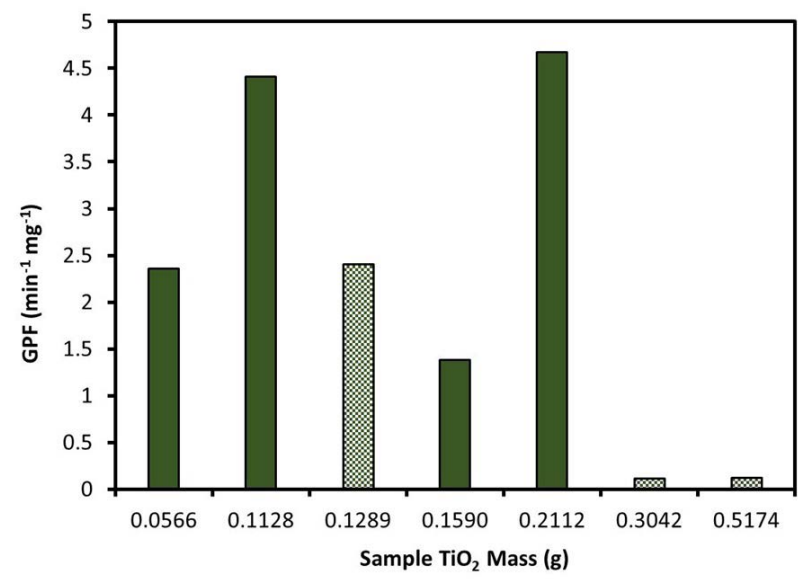

Figure 8: The green photocatalytic factor for benchmark (checkered) and revised (solid) samples. 
Citation: Glover JD, Horne AG, Crawford JD, Boyd JE (2017) Improved Fabrication Method for Porous PMMA-titania Composite Photocatalytic Materials. Int J Metall Mater Eng 3: 135. doi: https://doi.org/10.15344/2455-2372/2017/135

Page 5 of 5

samples lost $0.116 \mathrm{mg} \mathrm{TiO}_{2}$, whereas the revised-method samples lost only $0.081 \mathrm{mg} \mathrm{TiO}$. The GPFs calculated from the post- $1000 \mathrm{~h}$ activity and the corresponding $\mathrm{TiO}_{2}$ loss are shown in Figure 9. The GPF of the revised-method samples is $30 \%$ larger than that of the benchmark method.

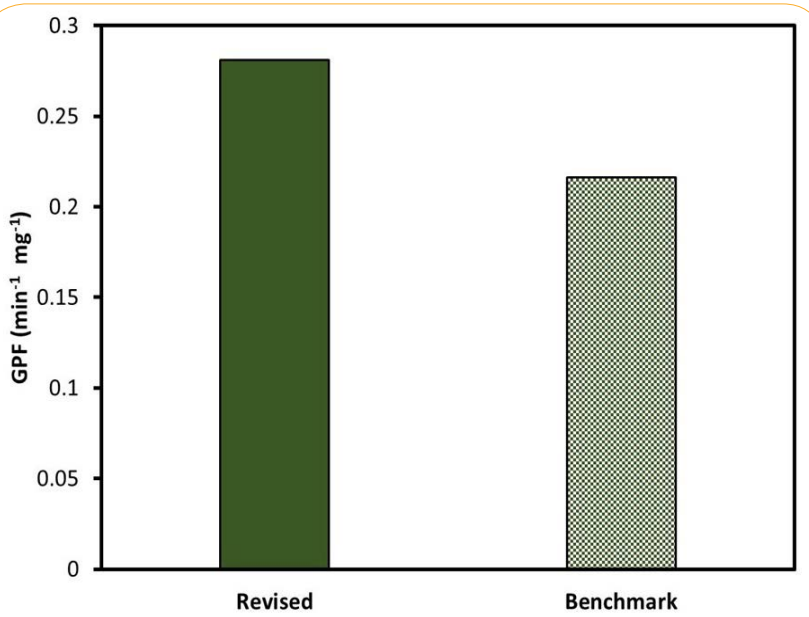

Figure 9: GPF for the revised and benchmark samples containing $0.113 \mathrm{~g}$ and $0.129 \mathrm{~g}$ of $\mathrm{TiO}_{2}$, calculated based on the methyl orange degradation rate constant after $1000 \mathrm{~h}$ of UV exposure and the mass of $\mathrm{TiO}_{2}$ lost during the $1000 \mathrm{~h}$ exposure time.

\section{Conclusion}

Incorporation of the $\mathrm{TiO}_{2}$ directly into the PMMA-acetone solution before coagulation with distilled water produces porous composite photocatalytic materials that use less $\mathrm{TiO}_{2}$ to achieve a comparable methyl orange degradation rate constant. When comparing samples of similar $\mathrm{TiO}_{2}$ content, the revised fabrication method reduces $\mathrm{TiO}_{2}$ use during sample preparation and makes it much easier to control the $\mathrm{TiO}_{2}$ content of the composite. $\mathrm{TiO}_{2}$ loss during photocatalytic use is also reduced with the revised fabrication process. The GPF provides a tool that incorporates both the amount of $\mathrm{TiO}_{2}$ lost during use and the photocatalytic activity when applying green chemistry concepts to photocatalytic systems. When comparing the GPF of samples with similar $\mathrm{TiO}_{2}$ content, the revised fabrication method is demonstrably improved over the benchmark method, providing a greener and better photocatalytic platform for photocatalytic water purification applications.

\section{Competing Interests}

The authors declare that they have no competing interests.

\section{Acknowledgements}

George Wetzel and Haijun Qian of Clemson University provided electron microscopy assistance. Sayed Hassan from the University of Georgia assisted with the $\mathrm{Ti}$ analysis for the assessment of the $\mathrm{TiO}_{2}$ lost from the composite surface. Degussa/Evonik graciously provided the $\mathrm{TiO}_{2}$ used in this study.

\section{Funding}

J. Glover was funded in part by a grant from the South Carolina Independent Colleges and Universities. Additional funding was provided by the Erskine Center for Environmental Stewardship.

\section{References}

1. Andronic L, Isac L, Miralles-Cuevas S, Visa M, Oller I, Duta A, et al. (2016) Pilot-plant evaluation of $\mathrm{TiO}_{2}$ and $\mathrm{TiO}_{2}$-based hybrid photocatalysts for solar treatment of polluted water. Journal of Hazardous materials 320: 469-478.

2. Malato S, Blanco J, Vidal A, Richter C (2002) Photocatalysis with solar energy at a pilot-plant scale: an overview. Applied Catalysis B: Environmental 37: 1-15

3. Loetscher LH, Carey JM, Skiles SL, Carey VM, Boyd JE (2009) TitaniaAcrylic Coil Reactor for Photocatalytic Water Purification and Sterilization. Industrial \& Engineering Chemistry Research 48: 4697-4702. Hiroyuki U, Shigeyoshi I, Hiroshi Y (1993) Photocatalytic Decomposition of Propyzamide Using $\mathrm{TiO}_{2}$ Supported on Activated Carbon. Chemistry Letters 22: 1995-1998.

4. Stewart BD, Andrews LG, Pelletier BS, Daly Jr CA, Boyd JE (2015) Porous PMMA-titania composites: A step towards more sustainable photocatalysis. Journal of Water Process Engineering 8: 179-185.

5. Mansilla HD, Bravo C, Ferreyra R, Litter MI, Jardim WF, et al. (2006) Photocatalytic EDTA degradation on suspended and immobilized $\mathrm{TiO}_{2}$ Journal of Photochemistry and Photobiology A: Chemistry 181: 188-194.

6. Jin L, Dai B (2012) $\mathrm{TiO}_{2}$ activation using acid-treated vermiculite as a support: Characteristics and photoreactivity. Applied Surface Science 258: 3386-3392.

7. Machado LCR, Torchia CB, Lago RM (2006) Floating photocatalysts based on $\mathrm{TiO}_{2}$ supported on high surface area exfoliated vermiculite for water decontamination. Catalysis Communications 7: 538-541.

8. Wang L, Wang X, Cui S, Fan X, Zu B, Wang C (2013) $\mathrm{TiO}_{2}$ supported on silica nanolayers derived from vermiculite for efficient photocatalysis. Catalysis Today 216: 95-103.

9. Ling CM, Mohamed AR, Bhatia S (2004) Performance of photocatalytic reactors using immobilized $\mathrm{TiO}_{2}$ film for the degradation of phenol and methylene blue dye present in water stream. Chemosphere 57: 547-554.

10. Carlson PJ, Pretzer LA, Boyd JE (2007) Solvent Deposition of Titanium Dioxide on Acrylic for Photocatalytic Application. Industrial \& Engineering Chemistry Research 46: 7970-7976.

11. Handy RD, Shaw BJ (2007) Toxic effects of nanoparticles and nanomaterials: Implications for public health, risk assessment and the public perception of nanotechnology. Health, Risk \& Society 9: 125-144.

12. Newman MD, Stotland M, Ellis Jl (2009) The safety of nanosized particles in titanium dioxide- and zinc oxide-based sunscreens. J Am Acad Dermatol 61: 685-692.

13. Szakal C, Roberts SM, Westerhoff P, Bartholomaeus A, Buck N, et al (2014) Measurement of Nanomaterials in Foods: Integrative Consideration of Challenges and Future Prospects. ACS Nano 8: 3128-3135.

14. Kumar A. (2012) Making a Case for Human Health Risk-based Ranking Nanoparticles in Water for Monitoring Purposes. Environ Sci Tech 46: 5267-5268.

15. Ratova M, Mills A (2015) Antibacterial titania-based photocatalytic extruded plastic films. Journal of Photochemistry and Photobiology A: Chemistry 299: 159-165.

This article was originally published in a special issue:

Photocatalysis Materials: Future Trends

Handled by Editor(s):

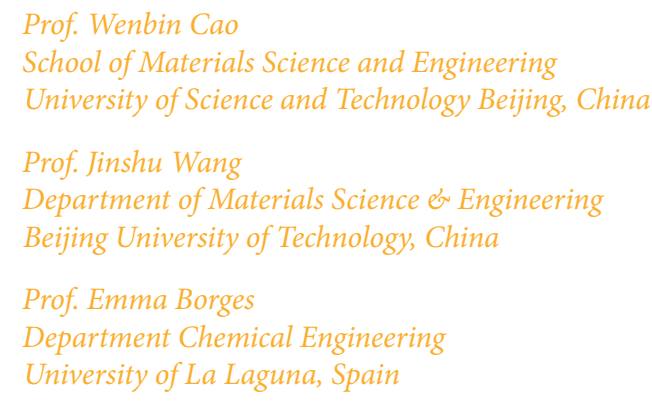

